\title{
One-dimensional Electron Systems
}

H.R. Zeller, Baden

H.R. Zeller is head of the general solid state physics group at the Brown Boveri Research Center in Baden (Switzerland).

\section{Introduction}

The major achievements in the field of 1-D conductors are that the existence of dimensionality effects has been demonstrated and that we have gained an understanding of the connections between molecular, structural and physical properties(1). This has already lead to a breakthrough in the chemistry and synthesis of organic charge transfer and free radical salts. Another major step foreward is that for the first time the full repertoire of solid state experimental techniques has been applied to such complicated systems and that it is possible to gain an understanding using relatively simple concepts.

Everybody who is somewhat familiar with mathematics is well aware of the fact that a problem is usually much easier to solve in one dimension than in two or three. As a consequence many famous problems in mathematical physics have been solved exactly in one dimension only. It also turns out that a generalisation of theorems obtained from the one-dimensional theory (1-D theory) to 2 and 3 dimensions is sometimes impossible or in other words strictly onedimensional systems show dimensionality effects ${ }^{(1)}$. These dimensionality effects are a direct consequence of the topoloogy of the interactions and have no counterpart in higher dimensions. There exists a large amount of theoretical work on this subject but with the exception of 1-D magnetic systems not much experimental work was available until recently.

Within a remarkably short time this situation has changed and today there is an extremely fruitful interaction between experiment and theory and a number of dimensionality effects have been shown to survive the nonideality of experimental systems.

A little more than a year ago Garito, Heeger and coworkers of the University of Pennsylvania Philadelphia announced the observation of superconducting fluctuations ${ }^{(2)}$ around $60 \mathrm{~K}$ in such a 1-D compound. The validity of both experiment and theory was subsequently questioned and the resulting controversy is still going on.

\section{Model systems}

Today the most important model systems are the mixed valency Ptsalts and the TCNQ (Tetracyanoquinodimethane) salts. In the mixed valency Pt-salts planar $\mathrm{Pt}$ complexes such as $\left[\mathrm{Pt}(\mathrm{CN})_{4}\right]$ are stacked to form linear and parallel strands of directly interacting $\mathrm{Pt}$ atoms. Partial oxidation gives rise to a partially filled band. The best known member of this class of compounds is

$$
\mathrm{K}_{2}\left[\mathrm{Pt}(\mathrm{CN})_{4}\right] \mathrm{Br}_{0} \cdot 30 \cdot 3\left(\mathrm{H}_{2} \mathrm{O}\right)^{(1)} \text {, }
$$
in short KCP.

TCNQ forms ionic salts with almost any cation in which the planar TCNQ molecules are arranged to form parallel stacks resulting in pseudo 1-D physical properties.

The best understood 1-D conductor today is KCP. KCP has a nearly free electron band structure. Peierls ${ }^{(3)}$ and Fröhlich ${ }^{(4)}$ have independently shown that a 1-D metal is inherently unstable with respect to a lattice distortion or charge density wave which creates an energy gap at the Fermi level and transforms the material into a semiconductor. This instability is a direct consequence of the 1-D topology of band structure resp. Fermi surface and has no counterpart in higher dimensions (a much weaker form exists in 2-D systems). Simple theory based on the Peierls-Fröhlich model predicts a semiconducting state at $\mathrm{T}=0 \mathrm{~K}$ and a transition into a metallic state at a critical temperature $T_{p}$. On the other hand it is well known that in true 1-D systems no phase transitions can occur at $T \neq 0 \mathrm{~K}$. In real systems a finite $T_{p}$ results from residual $3-D$ coupling. The PeierlsFröhlich instability in KCP has been studied in great detail by diffuse $x$ ray scattering, inelastic neutron scattering, optical and transport studies, NMR and other techniques. The lattice distortion resp. charge density wave connected with the Peierls instability was directly observed and the results can be summarized as follows: Without any fluctuations $T_{p}$ would be around $600-800 \mathrm{~K}$. Due to fluctuations KCP is still more or less metallic at room temperature but with greatly reduced electron mobility. Below $200 \mathrm{~K}$ the fluctuations are increasingly depressed by coupling to the 3-D system i.e. interstrand interactions and disorder. At $100 \mathrm{~K}$ and below the material is semiconducting.
Possibilities of high temperature paraconductivity

Fröhlich has noted that the charge density wave associated with the lattice distortion is free to move through the crystal and can carry a current and that scattering processes are effectively suppressed by the presence of an energy gap. In other words, the ground state of a 1-D metal is not an insulator but a superconductor. Lee, Rice and Anderson have shown that nonidealities such as impurities, disorder etc. introduce both lifetime effects and an energy gap in the exitation of the Fröhlich collective " surfing " mode. Hence instead of a $\delta$ function in $\sigma(\omega)$ at $\omega=0$ a peak at finite $\omega$ with finite width is predicted $(5)$. This behaviour is found in KCP. At $4.2 \mathrm{~K}$ a pronounced resonance in $\sigma(\omega)$ in the submicrowave region is found corresponding to oscillations of the pinned charge density wave.

While the situation is fairly clear in KCP the same is not true for TTF TCNQ (Tetrathiofulvalene - Tetracyanoquinodimethane) the best investigated TCNQ salt. Everybody seems to agree that TTF TCNQ has a room temperature dc conductivity of the order of $10^{3} \mid(\Omega \mathrm{cm})^{-1}$ which increases with decreasing temperature until around $60 \mathrm{~K}$ the material undergoes a metal to insulator transition. Typically $\sigma \quad(60 \mathrm{~K})$

$\sigma(300 \mathrm{~K})$

Penn group occasionally found ratios of up to $500^{(2)}$ a result which despite intensive efforts could not be reproduced in other laboratories. The Penn group claims that TTF-TCNQ undergoes a Peierls-Fröhlich transition in the vicinity of $60 \mathrm{~K}$ similar to KCP and that due to the higher degree of order in TTF-TCNQ as compared to KCP the pinning of the charge density wave is weaker. As a consequence the charge density wave can be thermally unpinned near $T_{p}$ and can give a dominant contribution to the observed dc conductivity, i.e. produce the giant peak. The Penn group stresses that such an effect would be extremely sensitive to sample perfection.

A group of scientists from Bell Labs and John Hopkins demonstrated that crystal imperfections can cause giant peaks in the apparent conductivity of such a material and that a classical four probe experiment is in general unreliable for highly anisotropic materials. M. Cohen and coworkers (Chicago) point out that attempts to find the Peierls distortion by $\mathrm{x}$-rays were unsuccessful in TTF TCNQ and that it is much more likely that the material becomes a ferroelectric at low temperature. 
On the other hand Heeger argues that the microwave measurements of his group demonstrate that the conductivity peak is real, that the Peierls distortion is not expected to be detectable with the $x$-ray techniques used up to now and that his optical experiments show the existence of a collective mode in $\sigma(\omega)$ at $\omega \approx 0$.

\section{Summary}

It is evident that true high temperature superconductivity in 1-D systems is not possible. The Fröhlich collective mode which is sort of a remnant of superconductivity has been observed in KCP and possibly in TTF TCNQ. Whether fluctuations into the superconducting state around $T_{p}$ really can give huge dc conductivities is still a matter of controversy both experimentally and theoretically. Although many workers in the field got attracted by the idea of high $T_{c}$ superconductivity, other aspects soon turned out to be at least as interesting. In my opinion the real achievement has been to open up a new area of solid state physics by demonstrating that by applying the full spectrum of solid state physics and not just standard materials research techniques, systems of the complexity of KCP and the TCNQ salts can be understood.

REFERENCES
1. H.R. Zeller, Advances in Solid State Physics,
Vol. 13, p. 31. Pergamon, Vieweg (1973).
2. L.B. Coleman, M.J. Cohen, D.J. Sandman,
F.G. Yamagishi, A.F. Garito and A.J. Heeger,
Solid State Commun. 12, 1125 (1973).
3. R.E. Peierls, Quantum Theory of Solids, p. 108.
Clarendon Press, Oxford (1955).
4. H. Fröhlich. Proc. Roy. Soc. A 223, 296 (1954).
5. P.A. Lee, T.M. Rice and P.W. Anderson, Solid
State Commun. 14, 703 (1974).

\title{
The Second Europhysics Conference of the Section of Macromolecular Physics
}

\author{
Sorrento, Italy, 1-3 May 1974
}

Postponed from October 1973 to May 1974, due to the epidemia in Naples, the Conference gathered 140 physicists in an unusually rainy Sorrento.

It was devoted to a highly controversial topic: The state of order in amorphous polymers.

$\mathrm{X}$-ray and electron diffraction patterns, together with some speculations on the dense packing of amorphous polymers compared with the crystalline state, has led to the idea that some local order, characterized by a parallel arrangement of chains in a liquid crystal fashion should exist. Illustrative models like the bundle model or the fringed micelles model have been proposed. For a quantitative approach however, only two extreme and contradictory models are available for comparison with experiments:

- The meander model of Pechhold and Blasenbrei which starts from the crystalline order and introduces well defined defects and superstructures to calculate a thermodynamically stable state compatible with the macroscopic isotropy and with the morphological and dynamic properties of polymer melts.

- The random coil model which extrapolates the gaussian statistics of isolated molecules in solution to the densely packed interpenetrating chains in the solid.

Papers explicitly dealing with the meander model mostly treated the formal development of the model in order to take into account new morphological and dynamic experiments (Pechhold, Hauber, Anthony). Optical transforms of ordered models including the meander model have been shown to give patterns closely resembling the electron diffraction patterns of some amorphous polymers (Bodor).
The most pertinent experimental contributions dealt with techniques appropriate to the direct detection of order at a scale of the order of about $30-50 \AA$.

Neutron scattering experiments on fully deuterated chains dispersed in a matrix of the same protonated polymer have been reported by one French and one German team (Janink, Kirste). They both conclude that not only is the radius of gyration of a chain in the amorphous bulk equal to that in a $\theta$ solvent (a prediction of the random coil model), but also that the scattering envelope fits the gaussian distribution down to $30 \AA$.

The study of density fluctuations by light and X-ray scattering (Wendorff, Ruland), as well as anisotropy fluctuation by anisotropic light scattering (Dettenmaier), above and below the glass transition temperature lead to the conclusion that no "frozen " density fluctuation, as would be expected from regions of local order, is needed to explain intensities and angular dependences which can be well understood in terms of normal thermal fluctuations.

A criticism of the spurious short range peaks introduced by termination errors in the calculation of the radial distribution function by Fourier Transform of the electron diffraction patterns has called the attention to possible misinterpretations (Voigt-Martin).

The conclusion of one paper on NMR line shape analysis of polyethylene melts is the existence of bundles with life times smaller than $10^{-4}$ seconds (Gölz). This result must, however, be treated with caution since an apparent superposition of lorentzian lines can arise from a non-exponential autocorrelation function as produced by a number of possible causes, such as normal entanglements.

If the general impression coming from these experiments is that order does not extend over more than a few angströms, it is fair to add that the "meander school" has claimed that some of this experimental evidence (such as the neutron scattering results) can equally well been explained by the meander model.

Other papers, less directly linked with this controversy, were concerned with :

- The calculation of vibrational modes in chains with well defined types of geometrical or stereochemical defects (Zerbi) and the determination of rotational isomerism in the solid.

- The role of short range order in rubber elasticity.

- The thermodynamics of the amorphous and glassy states incorporating an order parameter.

One session was devoted to the quite different order present in mesomorphic phases of block copolymers. Exciting new possibilities are opened by the ability to obtain monodomain samples. Indirect evidence for disorder in amorphous polymers arises from the lack of intrinsic optical anisotropy of cylindrical microphases $150 \AA$ in diameter (Keller). The existence of spherical microphases in block copolymer cubic microphases should be re-examined in the light of the quite different cubic structures of soaps and lecithines (Luzzati).

If the conference did not succeed in reaching a final conclusion on the state of order in amorphous polymers, the presentation of a series of new experiments and the confrontation of points of view has certainly contributed a step in the direction of a widely accepted model of polymer solid.
G. Weill, Strasbourg 